\title{
Analysis of Pre-Service Science Teachers' Heuristic Reasoning Processes about Hydrogen Bonding
}

\author{
Gülen Önal Karakoyun¹, Erol Asiltürk ${ }^{2 *}$ \\ ${ }^{1}$ Program of Chemistry Technology, Muradiye Vocational High School, VanYüzüncü Yıl University, Muradiye, Van, Turkey \\ ${ }^{2}$ Department of Science Education, Faculty of Education, Firat University, Elaziğ, Turkey \\ *Corresponding author: ecil@,firat.edu.tr
}

\begin{abstract}
The purpose of this research was to determine the heuristics used by pre-service science teachers in understanding the details of hydrogen bonding. The reasoning processes demonstrated were evaluated based on ten heuristic models suggested by Talanquer (2014). Phenomenographic assessment of the 30 participants indicated that all ten heuristics were utilized to make interpretations about hydrogen bonding. It was found that most students used short-cut strategies rather than efficient analytical reasoning processes. A total of 12 answer patterns were determined based on the answers of the participants. The percentage of students who gave the correct answer was low. The frequency sequencing of participants' heuristics demonstrated in this study was fluency, associative activation, recognition, one-reason decision making, attribute substitution, overconfidence, surface similarity, generalization, rigidity, and affect.
\end{abstract}

Keywords Science education, Hydrogen bonding, Educational psychology, Heuristics, Chemistry education

\section{INTRODUCTION}

One of the aims of science educators is to raise individuals who can use information effectively and logically (Vekli, 2020). In order to understand how individuals use information, reasoning processes should be examined in detail.

Many researchers have revealed that students have many mistakes and biases in reasoning processes related to science and chemistry topics (Ugras, 2018). One of these science/chemistry subjects that are incompletely or incorrectly interpreted by students is the subject of hydrogen bonding. Hydrogen bonding is a type of interaction between particles, and it has an essential place in chemistry, biochemistry, biology, and molecular biology. The effects of this bonding type are evident in many biotic phenomena. For instance, two DNA strains are connected through hydrogen bonding. Antibody and antigen bonding are also based on hydrogen bonding. Hydrogen bonding is efficient in the duplication process called transcription in protein synthesis. Water, which has a vital role in organisms, and many sugar molecules have hydrogen bonding.

Students need to have correct and robust insight about hydrogen bonding to grasp and interpret different types of biological phenomena which are of vital importance to organisms; they need to know the characteristics of hydrogen bonding to analyze different chemical phenomena such as boiling and dissolution as hydrogen bonding plays a significant role in these processes.

Researches on students' perceptions about hydrogen bonding indicate that they generally don't understand the process accurately and have a lot of inadequacies about the subject (Barker \& Millar, 2000; Cooper, Williams \& Underwood, 2015). It is important and crucial to make indepth analyses about the reasoning processes of students in science subjects; it will thus be possible to create and improve strategies that remove their misconceptions not only about the issue of hydrogen bonding but also other significant subjects in science.

Science educators who want to research and understand the nature of mistakes and biases in student reasoning processes on science subjects have recently started to show interest in various disciplines. There are many kinds of studies in various disciplines such as developmental psychology and cognitive science, which analyze individuals' judgment and decision-making processes in daily and social life from different perspectives (Connor \&

Received: 21 March 2020

Revised: 30 September 2020

Published: 28 November 2020 
Becker, 2003; Ferrari \& Dovidio, 2000; Hastie, 2001; Schwarz, 2004).

Based on these multi-dimensional research results, different theories are established to explain individuals' judgment and decision-making processes (Gilovich, Griffin \& Kahneman, 2002; Todd \& Gigerenzer, 2000; Ugras, 2018). One of these theories is the "Dual Process" theory, which involves essential information about individuals' intuitive judgment and decision-making (Evans, 2008; Evans \& Stanovich, 2013; Gigerenzer \& Goldstein, 1996; Ugras, 2018). This model's essential purpose is to explain individuals' reasoning about daily life, and it has recently become a frequently-used method in explaining students' reasoning about scientific issues. According to the dual-process theory, humans have two distinct types of reasoning processes: Type 1 and Type 2 . Type 1 processes are independent of cognitive ability and involve reasoning forms that move very fast without giving importance to working memory (Ugras, 2018). As there is no need for a specific effort to trigger Type 1 processes, they automatically occur (Stanovich \& West, 2000; Ugras, 2018). Type 1 processes are autonomous, involving learned strategies, and naturally formed reasoning processes (Stanovich \& West, 2000). Type 1 processes are about intuitive judgments. On the other hand, working memory is employed in Type 2 processes, which work slowly and successively (Talanquer, 2014). Practicing Type 2 processes requires extraordinary effort and deliberate intervention; these processes are about individuals' hypothetical, analytical, or reflective thinking ways (Maeyer, 2013; Ugras, 2018). Studies reveal that when an individual faces a new problem or situation, Type 1 processes are instantly triggered (Evans, 2008; Graulich, 2014; McClary \& Talanquer, 2011). Type 1 processes are responsible for various biases formed during reasoning processes (Ugras, 2018). Type 1 processes are considered short-cut reasoning strategies and are called "heuristics" (Graulich, 2014; Ugras, 2018). As heuristics shorten the information processing path, they ensure individuals to make decisions sooner than expected; they set some implicit rules about reasoning processes intended for solving a problem, and thus ease individuals' reasoning (Todd \& Gigerenzer, 2000).

Since heuristics are unique, fast, and frugal mental structures, individuals can make rational decisions when used heuristics consciously to solve problems they encounter in science topics. Thus, examining how students use heuristics in science subjects and researching methods that will enable them to use heuristics consciously will be beneficial in increasing the quality of science education.

The studies on the use of heuristics in the subjects of chemistry are relatively recent. Students' use of heuristic on the subjects of addition reactions, elimination reactions, chemical problem solving, acidity strength of molecules, chemical reactivity, chemical bonding theories, classification of chemical substances, structure-property relationships of molecules, and interpretation of IR and $\mathrm{H}$ NMR spectra have been investigated thoroughly until today (Cooper, Corley \& Underwood, 2013; Connor, Finkenstaedt-Quinn \& Shultz, 2019; Graulich, Hopf \& Schreiner, 2011a; Graulich, Hopf \& Schreiner, 2011b; Maeyer, 2013; Maeyer \& Talanquer, 2013; McClary \& Talanquer, 2011; Ugras, 2018). These studies reveal that students correctly and incorrectly answered questions without using the basic and important chemical knowledge because of the effects of heuristics. Any kind of model (such as the ten heuristics model suggested by Talanquer) or standard was not ever used in the researches mentioned above about chemistry subjects. Researchers independently determined and named heuristics in each of those studies. In addition to these critical studies, Talanquer has explained the frequently used heuristics in chemistry according to the employed cognitive processes and made an essential contribution to the literature by grouping these different heuristics under ten categories (Talanquer, 2014). The model of Talanquer is highly significant, and it provides a reference point for future studies in the field of chemistry. For instance, in Miller and Kim's study, on the use of heuristics by students in hydrogen bonding, the model proposed by Talanquer was used as the basis (Miller \& Kim, 2017). This research by Miller and Kim is the first and the only study that explains the use of heuristics in chemistry subjects according to the model proposed by Talanquer. As they did not use the interview technique in their research, they could examine only six of the ten heuristics. However, in the current study designed meticulously, interviews were conducted with students to make detailed analyses about the effects of 10 different heuristics in students' reasoning processes about hydrogen bonding. According to Talanquer's model, ten heuristics can be efficient in students' reasoning processes in chemistry subjects. These heuristics are Associative activation, Fluency, Attribute Substitution, One-Reason Decision Making, Surface Similarity, Recognition, Generalization, Rigidity, Overconfidence, and Affect (Talanquer, 2014). In his theoretical study, Talanquer explained each of these ten heuristics and their specific examples of the chemistry field (Talanquer, 2014).

The purpose of this study is to determine and explain the heuristics used by pre-service science teachers in the subject of hydrogen bonding; the study is based on the model suggested by Talanquer (2014). 


\section{METHOD}

\subsection{Sample}

This study was conducted during the spring semester in the 2018-2019 academic year in Turkey's public university. A total of 30 teacher candidates at $2 \mathrm{nd}$, 3rd and 4 th grades in the Faculty of Education Science Teaching program have voluntarily participated in the research. Sixteen of the participants were male, while 14 were female teacher candidates. Success rates of students in General Chemistry I and General Chemistry II were considered while determining the ones to participate in the study. One-third of the participants were unsuccessful; one-third were midlevel successful, while one-third were highly successful in these specific classes. The participants' actual names were not used in the study; they were encoded as S1, S2, S3, and S4...

\subsection{Research Methods, Instruments and Procedure of Interview Protocol Development}

In this study, phenomenographic research methodology, one of the qualitative research methods, was preferred to investigate students' heuristic use in the subject of hydrogen bonding. Phenomenography, used in educational researches for a long time, is defined as: "An empirical study whose aim is to discover the quantitatively different ways in which people experience, realize and understand various aspects of a phenomenon" (Akerlind, 2005; Didis, Ozcan \& Abak, 2008). Phenomenography is a widely accepted methodology in education research. It is used for representing the differences in the understandings of individuals and how they perceive a single concept in different ways (Didis, Ozcan \& Abak, 2008; Wihlborg, 2004). Generally, interviews are done for gathering detailed information in phenomenographic research studies; this is why interviews are held with participants to accurately determine their reasoning about hydrogen bonding and identify the heuristics employed in this process. Students were required to answer a question developed by Miller and Kim (2017) containing the Lewis demonstration of acetic acid. The structural demonstration of acetic acid is presented in Figure 1.

Each atom in the molecule is numbered explicitly in the question involving the demonstration of acetic acid's structure. Students were asked to answer the question of "With which of the numbered molecules can the water molecule form the hydrogen bonding?" Miller and Kim define this question type as a multiple-select format assessment item that requires the 'selection of all valid choices' (Miller \& Kim, 2017). During the interviews of this research, students were given 2 minutes to answer the question. In the relevant literature, it is stated that the impacts of intuitive judgment and decision-making are higher in the states where the time is limited (McClary \& Talanquer, 2011); for this reason, the time given for this step during the interviews was limited. Then, the participants were required to explain the reasons for their

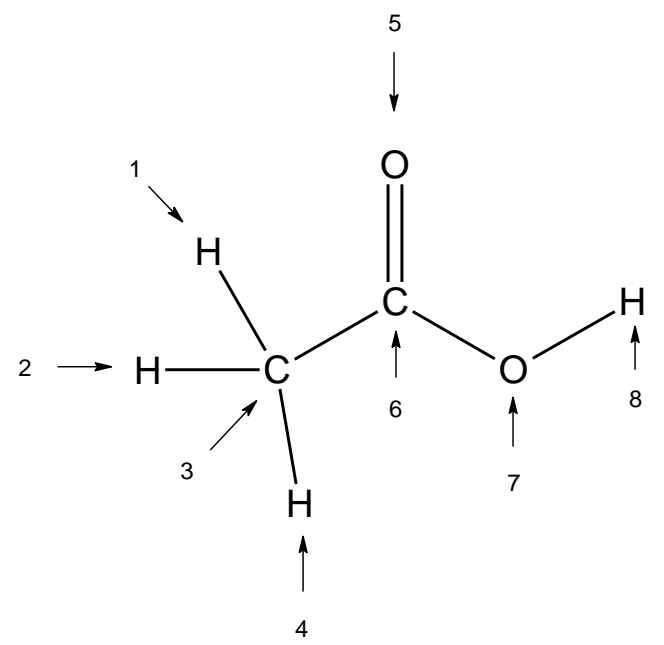

Figure 1 Structural demonstration of acetic acid. Each atom in the structure is specifically numbered

answers in detail. Time-limitation was not conducted at this stage of the process. Some additional questions were asked to the participants before and after they answer the question; the purpose of these questions was to determine if all of the heuristics suggested by Talanquer, especially 'rigidity, overconfidence and affect' heuristics, took part in the answering process. Interviews were recorded both audio-visually, and then these records were transcribed verbatim; interview transcripts for each student were prepared. Heuristic reasoning has been identified and encoded based on the analyses of interview transcripts. Previous research studies that contain interview procedures were also considered during the encoding process of the current study (Miller \& Kim, 2017; Ugras, 2018).

To ensure the inter-rater reliability, 8 randomly chosen interview transcripts (approximately 25\%) were evaluated separately by the researcher and the consultant, and encodings were completed. Results obtained from the two raters were compared, and encodings were revised to ensure $90 \%$ coherence. After catching the coherence level, all of the remaining interview transcripts were evaluated and encoded by herself. Ten heuristics suggested by Talanquer (2014) were used to create an encoding scheme about heuristics. The heuristics encodings, except rigidity, overconfidence, and affect, were carried out by associating students' specific statements about the solution of the question with heuristics. Specific student statements that are the basis of encodings are presented in the results section. The procedure, explained below in detail, was followed to identify and encode the heuristics about rigidity, overconfidence, and affect heuristics.

Rigidity: The steps followed for researching rigidity heuristic in this study started with asking the participants a few questions before directing the question about acetic acid. The students were required to answer the questions: "Do you have an absolute judgment/bias about the subject of hydrogen bonding?" "For instance, do you have any approaches such as: In the subject of hydrogen bonding, I 
have some specific judgments/reasoning that I will never change whatever the question is. I always make evaluations and solve problems by using these judgments/reasoning processes." Answers of participants to this question were carefully analyzed.

On the other hand, it was carefully analyzed during interviews if participants actually used their previous strategies and solved the problem accordingly, and if they were flexible while answering questions. Three specific statements were determined and encoded as the indicators of rigidity heuristics; if participants solved the problem based on the encoded statements they mentioned and had no flexibility, they would fit into this category. Statements encoded as rigidity heuristics were: "No matter what the question about hydrogen bonding is, I will think that $\mathrm{F}, \mathrm{O}$, $\mathrm{N}$ and hydrogen atoms form hydrogen bond; I will make an evaluation based on this consideration", "No matter what the question about hydrogen bonding is, I will think that all hydrogen atoms form hydrogen bonds; I will make evaluations based on this consideration" and "No matter what the question about hydrogen bonding is, I will think that only oxygen atoms form hydrogen bonds; I will make evaluations based on this consideration".
Overconfidence: The way followed for researching the effects of overconfidence in participants started with directing a question to the participants before asking/showing them the question about acetic acid: "Do you believe that you can give the correct answer to a question about the subject of hydrogen bonding? What is your self-confidence level; how would you rate your selfconfidence on a scale from 1 to 10 (1 is the lowest, 10 is the highest rate)?" Right after asking/showing the question about acetic acid, before students started solving the problem, they were directed another question: "Do you believe that you can give the correct answer to this question. What is your self-confidence level; how would you rate your self-confidence on a scale from 1 to 10 ( 1 is the lowest, 10 is the highest rate)?" Finally, a question was directed to participants after they solved the problem: "How much do you confide in your answer? How would you rate your confidence in your answer on a scale from 1 to 10 (1 is the lowest, 10 is the highest rate)?" Overconfidence heuristics is encoded when the students scored all three questions as 8,9 , or 10 . Students with such answers generally had statements such as: "I trust in myself, I gave/will give the correct answer".

Table 1 Answer patterns obtained from the question

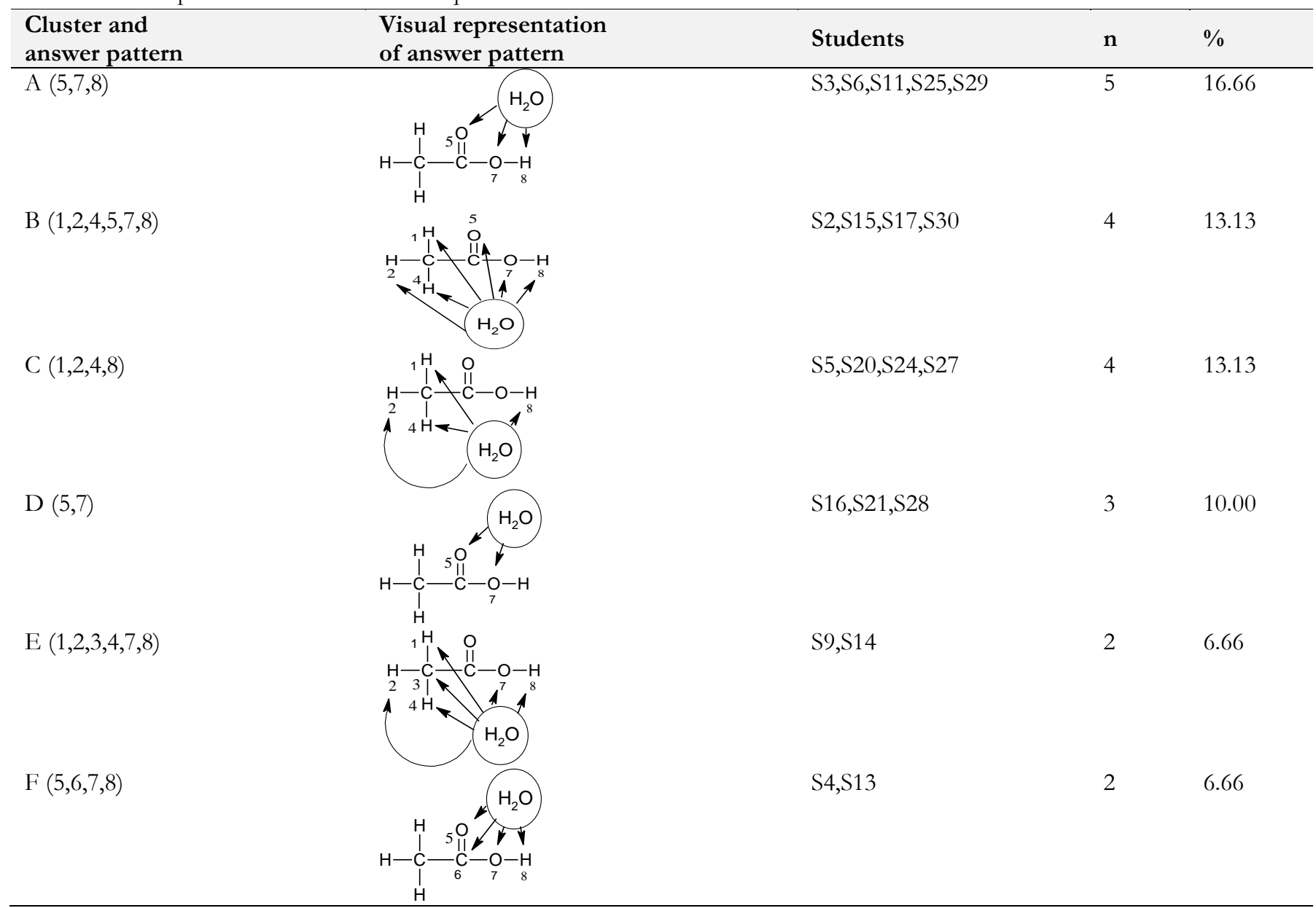


Table 1 Answer patterns obtained from the question (Continued)

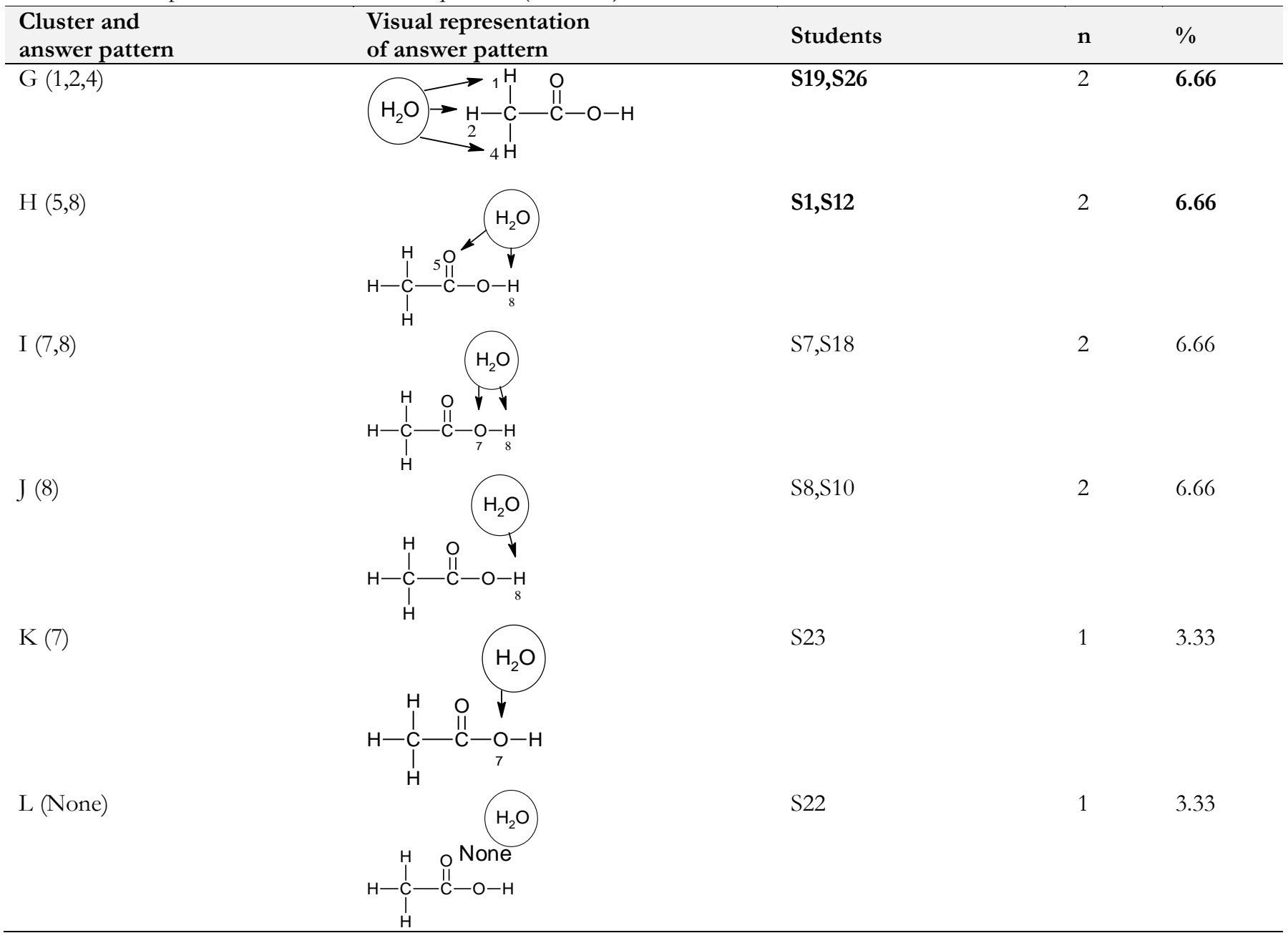

Affect: In order to research the effect of affect heuristic on the subject of hydrogen bonding, a question was directed to participants before asking/showing them the question: "How would you feel when you think about the subject of hydrogen bonding; throughout your education, did you have any experience about the subject that may positively or negatively affect you? If so, does it still have any effect on you?" On the other hand, after asking/showing the question, the participants were required to answer the question: "How do you feel now once you have seen the question?" Affect heuristic is encoded according to participants' answers in the cases when it was determined that they have positive or negative perceptions because of their past experiences.

\section{RESULT AND DISCUSSION}

In this research, interviews were held with participants to evaluate how they perceive hydrogen bonding in the context of a structural demonstration. During the interview processes, participants were required to answer the question involving Lewis's structural demonstration of acetic acid: "Which of the atoms in acetic acid can form a hydrogen bond with water molecule?" After the carefully followed answer-analysis process, a total of 12 different answer patterns were obtained. These 12 different answer patterns and the code names of students who gave these answers were presented in Table 1. The number of students in each pattern and the percentage and the visual representation of each answer pattern was also presented in Table 1.

Numbers under the title of the cluster and answer pattern in Table 1 stand for the atoms numbered on acetic acid's structural demonstration (Figure 1). As shown in Table 1 , only $16.66 \%$ of students gave the correct answer (Cluster A). According to the literature results, the number of students who comprehended the subject of hydrogen bonding correctly is relatively few; this result is similar to the current study's findings (Taagepera et al., 2002; Villafane et al., 2011). For example, according to the study by Villafane et al., the number of students who understood the subject was few, and students had some misconceptions about hydrogen bonding (Villafane et al., 2011). On the other hand, in their study, Miller and Kim obtained a similar result and determined that the number of students who understood the subject correctly was few (Miller \& Kim, 2017). 
Table 2 Heuristic codes and summaries of student statements

Heuristic Code
Associative Activation
Use of the existent mental constructions to
fill in the blanks
Fluency
Use of easily accessible cues
Attribute Substitution
Substitution of the original question with a
simpler one

One-Reason Decision Making

Simplification of reasoning with the use of a single cue or factor

Surface Similarity

Simplification of reasoning by assuming that similar objects can be chemically

comparable

\section{Recognition}

Use of a specific aspect as the decision cue Generalization

Generalization of learned model or rules

Rigidity

Reasoning that is not flexible and creative

\section{Overconfidence}

Exceeding the actual accuracy because of the self-confidence in decision-making

processes

Affect

Positive or negative senses

\section{Summary of Student Statements}

All hydrogen in the molecule form hydrogen bonds (hydrogen bond equals to all hydrogen)

Full octet should be formed

Water should form be formed

I have searched for a dual bond atom in the molecule

I have searched for a part similar to water in the molecule

I have searched for hydrogen attached to fluorine, oxygen or nitrogen

Substitution of the original question with these questions:

Which atoms can form water?

Which atoms can undergo condensation reaction?

Which bonds undergo addition reactions?

Which part of the molecule is similar to water?

Between which atoms is there a dual bond?

Carbon cannot form a hydrogen bond

Oxygen cannot form dual bond

Atoms with dual bond cannot form hydrogen bond

This aspect of acetic acid is similar to water. There is hydrogen bond in water; then, atoms that form the parts of acetic acid that are similar to water can form hydrogen bond. Water molecule doesn't involve dual bond; this is why water molecule can form hydrogen bond with the atoms in acetic acid that are similar (atoms only attached with only single bond)

$\mathrm{H}, \mathrm{F}, \mathrm{O}, \mathrm{N}$ rule

Octet rule is prevalent while hydrogen bond is formed

Hydrogen bonding is a chemical reaction (condensation)

The bond orders are taken into consideration while hydrogen bond is formed

I think that only F, O, N and hydrogen atoms can form hydrogen bond; I will make evaluations on this basis

I think that all hydrogen atoms in a molecule can form hydrogen bond; I will make evaluations on this basis

I think that only oxygen atoms can form hydrogen bond; I will make evaluations on this basis

I absolutely solved/will solve the question correctly

My self-confidence level is between 8 and 10

I like/don't like the subject of hydrogen bonding; positive/negative sense
According to the researches in the literature about hydrogen bonding, the most frequent misconceptions of students are: "All hydrogen can form hydrogen bonds", "Hydrogen bond is a covalent bond", "Hydrogen bonds occur inside the molecules rather than occurring between them", "Covalent bonds are broken during phasetransition", "Hydrogen bond is any bond that involves hydrogen", "All polar molecules can form hydrogen bonds" and "All compounds containing nitrogen, oxygen, and fluorine together with hydrogen form hydrogen bonds, regardless of whether the hydrogen is bound to these atoms (Duis, 2011; Henderleiter et al., 2001; Tan \& Treagust, 1999; Villafane et al., 2011; Pérez et al., 2017). In this study, whose basic purpose is to analyze the use of heuristics by students in the subject of hydrogen bonding, a multipleselect format assessment item is used for analyzing the possible interactions of a molecule at the level of atoms; this selection of assessment also enabled the researcher make direct observations about some misconceptions of students. For example, the misconception that "all hydrogen can form hydrogen bonds" was determined in previous research. It is also reflected in this study in Cluster C (atoms numbers 1, 2, 4, and 8). This pattern is formed based on participants' answers, who have chosen the entire hydrogen atoms rather than choosing the other atoms in the molecule. As shown in Table 1, the percentage of students in this answer pattern is $13.13 \%$. Inclusion of answer patterns based on students who have chosen the hydrogen atoms exclusively, but not each hydrogen (Cluster $\mathrm{G}$ and J), would increase this ratio to $26.66 \%$.

The structural/visual presentations of the answer patterns in each cluster are given in Table 1. Cluster B 


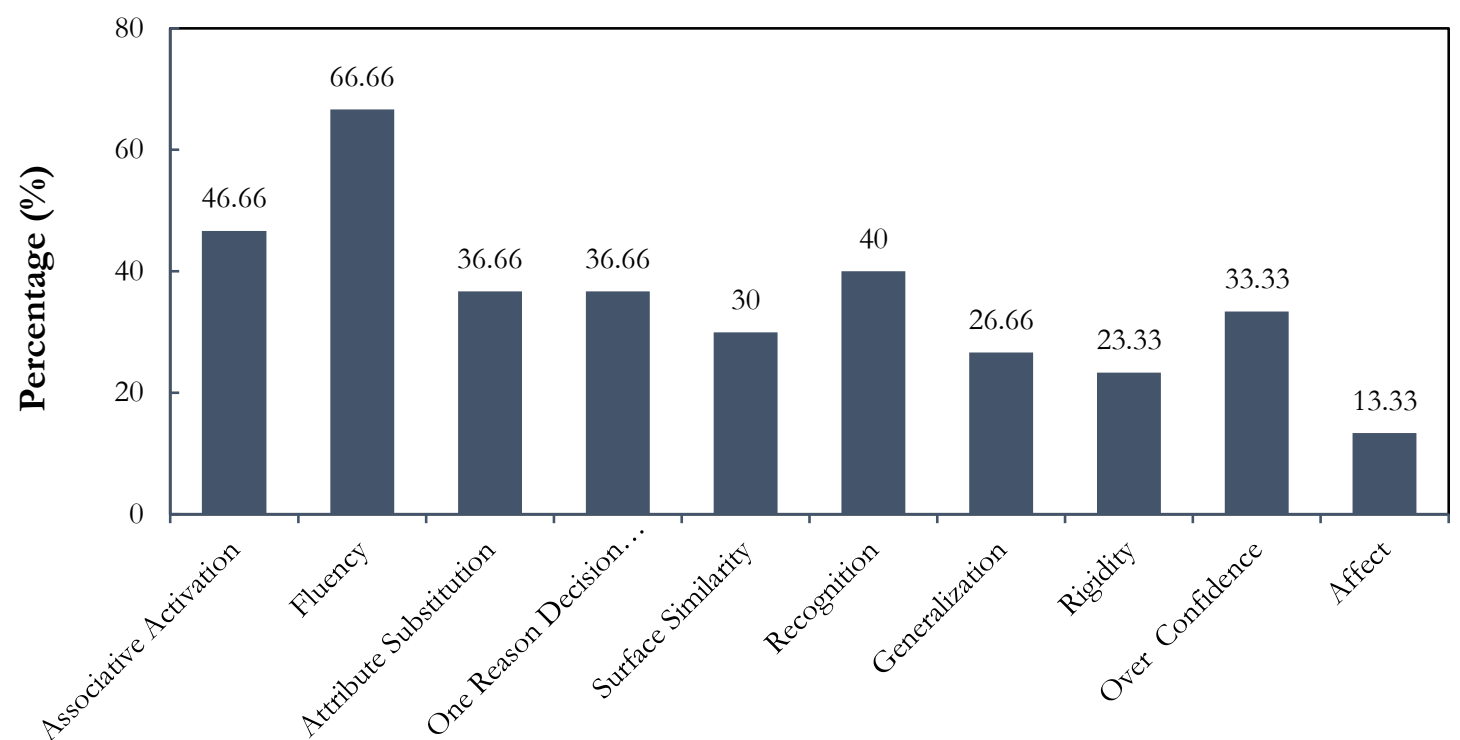

Heuristics

Figure 2 Graphical presentation of heuristic usage percentages

$(13.33 \%)$ and Cluster C $(13.33 \%)$ are the most preferred selections after Cluster A (16.66\%), which is the correct answer. In Cluster B, all of the atoms except for the two carbons in the structure are involved in the calculation. In Cluster C, only all of the hydrogen in the structure is involved in the calculation. In Cluster D $(10.00 \%)$, only oxygen atoms are selected. In Cluster E (6.66\%), all of the atoms except for the ones with double bonds (carbon and oxygen) are selected. In Cluster G (6.66\%), all hydrogen except for the one attached to oxygen is involved in the calculation. These answers are related to using strategies based on simple rules such as "all atoms except for carbon" and "only hydrogen atoms". The use of strategies based on a specific rule is an indicator of heuristics' role in answering processes (Miller \& Kim, 2017).

For this reason, student answers have been analyzed in terms of heuristics used. Codes were made by associating the specific statements in the participants' responses with ten heuristics. Summaries of student statements that provide a basis to encodings about these ten heuristics are presented in Table 2.

Hydrogen bonding is generally defined as the attractive forces between hydrogen attached to an electronegative atom and another electronegative atom. To decide which atoms in a compound can form a hydrogen bond with water molecule, it is necessary to consider the factors of electronegativity and polarity. In the question, students were required to evaluate the relationship of electronegativity between two atoms. They were also required to accurately state which atoms in a compound (acetic acid) can form a hydrogen bond with water according to their assessment of the electronegativity relationship. This is the implied target attribute. However, in this study, it is determined that participants could not accurately evaluate the target attribute or evaluate some other attributes instead of the target one. To express the results visually, heuristic usage percentages are given in Figure 2 as a graphical representation.

Participant names (pseudonyms of the participants) that have used the relevant heuristics are as follows; Associative Activation: S1, S4, S5, S8, S10, S12, S13, S19, S20, S22, S23, S24, S26, S27; Fluency: S1, S2, S3, S4, S6, S7, S8, S9, S10, S11, S12, S13, S14, S15, S17, S18, S23, S25, S29, S30.; Attribute Substitution: S1, S4, S7, S8, S9, S10, S12, S13, S14, S18, S23.; One Reason Decision Making: S2, S7, S9, S14, S15, S16, S17, S18, S21, S28, S30.; Surface Similarity: S1, S4, S7, S8, S10, S12, S13, S18, S23.; Recognition: S2, S3, S6, S11, S15, S16, S17, S21, S25, S28, S29, S30.; Generalization: S4, S5, S13, S19, S20, S21, S22, S26.; Rigidity: S2, S20, S21, S25, S27, S28, S30.; Overconfidence: S2, S6, S11, S15, S17, S20, S21, $\mathrm{S} 24, \mathrm{~S} 29, \mathrm{~S} 30 . ;$ Affect. S1, S8, S23, S29.

It is crucial to determine the points specifically required in a question accurately. This crucial step may sometimes be perceived as a straightforward process. However, in this research, according to the reasoning of participants employed while solving the problem, heuristics revealed that heuristics affected their interpretations, and thus there are differences in participant statements about the target attribute implied in the question. The reason for these differences is the impact of the attribute substitution heuristic; through this heuristic, complications are decreased by unconsciously ignoring information that is, in fact, crucial for solving problems (Ugras, 2018). This study determined that heuristics such as fluency, generalization, and surface similarity trigger the heuristic of attribute substitution; this heuristic caused 11 of the participants $(36.66 \%)$ to substitute the original problem with another one. For example, the statement of "Hydrogen bonding is a reaction of condensation" results from the generalization heuristic's effect. Due to the effect of this reasoning, 
attribute substitution heuristic is triggered, and students, thus, simplified the original question and changed it with a much simpler form: "Which bonds or atoms in acetic acid can undergo condensation reaction?" These students have also focused on this simple question instead of the target attribute in the following processes. In this study, it is determined that with the effect of attribute substitution, students focused on answering these questions instead of the original one: "Which atoms can form water?", "Which atoms can undergo condensation reaction?", "Which bonds undergo condensation reaction?", "Which parts of the molecule are similar to water?" and "Which atoms have a dual bond?"

The heuristic of attribute substitution, which caused students to focus on the questions mentioned above instead of the target attribute, is triggered and supported by other heuristics such as fluency and surface similarity. Heuristics usually trigger one another; thus, more than one heuristic is efficient in the decision-making processes (Ugras, 2018). This study is in line with the literature statements; participants in this study used multiple heuristics in answering processes. The problem-solving method of the participant S7 is an example of the use of multiple heuristics;

S7: Atoms that can form a bydrogen bond with water are the ones number 7 and 8 .

Interviewer: Will you please explain the strategy you used for answering the question?

S7: I was a little surprised when I first saw the question as the question is interesting and unusual for me; this is why I had no idea about how to solve the problem at the beginning. I started to carefully analyze the structural demonstration of acetic acid on the chance of finding a solution. I thought that there is a cue in this demonstration that I can use as a starting point, and it can help me solve the problem. On the one hand, I was thinking about these; on the other, I analyzed the shape of the molecule to find a cue. Then $\mathrm{OH}$ group in the molecule attracted my attention. I started to think if I can use the -OH group as a cue. This part of the molecule, namely -OH group, was similar to water. This similarity made me think about the structure of the water molecule. I was sure that water has bydrogen bonding. Since water can form a bydrogen bond, then molecules that structurally involve groups similar to water can form bydrogen bonds through these parts. Based on this reasoning, I reanalyzed the acetic acid molecule to see if any other parts in it are similar to water. The only part similar to water was $-\mathrm{OH}$ group formed by the atoms number 7 and 8. As I thought that hydrogen (atom number 7) and oxygen (atom number 8) atoms in this part could form a bydrogen bond, the answer is the atoms number 7 and 8 according to me.

The use of $-\mathrm{OH}$ group on the acetic acid molecule as an easily accessible cue indicates the efficiency of fluency heuristic in the reasoning process of participant S7. The heuristic of surface similarity also played an essential role in this process. The assumption that chemical compounds that are similar in terms of the structural demonstration are the members of the same category and believe that these types of compounds have similar features and behaviors result from the impact of surface similarity heuristic (Talanquer, 2014). In this context, it can be said that the reasoning of participant S7 (Since water can form a bydrogen bond, then molecules that structurally involve groups that are similar to water can form hydrogen bond through these parts") is a result of this heuristic's impact. Participant S7 looked for a part on the acetic acid molecule that is similar to water. S/he thus changed the target attribute and focused on finding an answer to another question (Which part of the molecule is similar to water?). This type of behavior is a result of the impact of attribute substitution. The same student trusted in a single reason (Only water-like parts of the molecule form hydrogen bond) in the process of answering the question and $\mathrm{s} /$ he solved the problem based on this criterion; so, it can be said that one-reason decision-making heuristic was also efficient in the student's reasoning process. Based on these reasons, as they were efficient and dominant in the reasoning process of participant S7, fluency, surface similarity, attribute substitution, and onereason decision-making heuristics are encoded as the ones used by the student.

Participants frequently used surface attributes unconsciously, similar to the example mentioned above. Surface attributes are easily accessible cues, and their unconscious use is also related to the heuristic of fluency. Students' tendency towards easily accessible information is considered a result of the fluency effect (Talanquer, 2014). Fluency effect manipulates strategies or cues used for a duty; thus, it is the most common heuristic that plays a role in decision-making processes. Besides, it enforces the other heuristics (Ugras, 2018). In this research, it is determined that the fluency effect played a significant role in the decision-making processes of 20 participants while solving the problem (66.66\%, the most commonly used heuristic). Some participants used double bonds or $-\mathrm{OH}$ groups in the acetic acid molecule as an easily accessible cue; this process results from the fluency heuristic. Along with the fluency heuristic, some other heuristics triggered and enforced one another in these processes. The problemsolving method of participant S4 is an example that shows the occurrence of many other heuristics because of fluency.

S4: Atoms that can form a bydrogen bond with water are numbers 5, 6, 7, and 8 .

Interviewer: Will you please explain the strategy you used for answering the question?

S4: ........ at that point, the double bond on the molecule attracted my attention. I suddenly remembered the other reaction when I was thinking about the double bond. I do not know if it is correct or reasonable, but I think that the bydrogen bond formation is an addition reaction; this is why I started thinking about addition reactions. As much as I remember, compounds with double bonds could undergo an addition reaction. On the other hand, water was formed as a by-product of addition reactions. The question was about 
the atoms that could form a bydrogen bond with water. Water bonds to atoms that form the dual bond on acetic acid (atoms number 5 and 6) to form bydrogen bond. As a result of this addition reaction, namely the formation of the bydrogen bond, some atoms should be separated from the structure of acetic acid and form water. $-\mathrm{OH}$ group connected to carbon atom number 6 is similar to water. Almost certainly, the $\mathrm{OH}$ group causes water formation by separating from the molecule during the addition reaction. Another water molecule could have formed a bydrogen bond with the oxygen and bydrogen atoms (atoms number 7 and 8) in -OH group to separate this $-\mathrm{OH}$ group. I believe that atoms in $-\mathrm{OH}$ group can form hydrogen bonds because this group is water-like. I thought that if water can form a hydrogen bond, then molecules that involve water-like groups in their structure can form bydrogen bond through these parts. I am not sure if my explanations are correct, but I decided based on these thoughts I mentioned above. I checked to see if there is any other dual bond in the molecule before making my final decision. There was no other dual bond. As a result, in my opinion, the answer is atoms numbers 5, 6, 7, and 8.

As shown above, participant $\mathrm{S} 4$ used the double bond and $-\mathrm{OH}$ group as a cue in the answering process; this use is an example of the result of fluency heuristic. The student's approach is built based on the perception that "hydrogen bonding is an addition reaction," which indicates that the generalization heuristic was also efficient in the process. The reasoning of "Water is formed in the process of hydrogen bonding" implies the heuristic of associative activation. Instead of the target attribute, the student-focused on finding answers to some other questions such as "Between which atoms is there dual bond" and "Which part of the molecule is similar to water?" Focusing on some other and more straightforward questions result from the impact of the attribute substitution heuristic. The student thought that $-\mathrm{OH}$ group could also form hydrogen bonds similar to water; this has resulted from the surface similarity heuristic impact. As a result, the heuristic of fluency, which was efficient in the first approach of participant S4, triggered the heuristics of generalization, associative activation, attribute substitution, and surface similarity.

Some of the students stated that they specifically focused on $\mathrm{H}, \mathrm{F}, \mathrm{O}$, and $\mathrm{N}$ atoms in problem-solving processes; when they were asked about the reason behind this, they said that $\mathrm{H}, \mathrm{F}, \mathrm{O}$, and $\mathrm{N}$ atoms would appear in their mind as soon as they heard about hydrogen bonding and they somehow related these atoms with this type of bonding. The researcher asked these students about the relationship between $\mathrm{H}, \mathrm{F}, \mathrm{O}$, and $\mathrm{N}$ atoms and hydrogen bonding. They stated that when the topic of hydrogen bonding was mentioned in chemistry classes, these atoms would commonly be mentioned; they were thus familiar with them, and they had the perception that only $\mathrm{H}, \mathrm{F}, \mathrm{O}$, and $\mathrm{N}$ atoms could form a hydrogen bond. The participants said that this perception is called " $\mathrm{H}, \mathrm{F}, \mathrm{O}, \mathrm{N}$ rule". Individuals' tendency to trust in the information that is easier to recall from the memory is considered as the effect of recognition heuristics (Talanquer, 2014); thus, the students' trust in $\mathrm{H}, \mathrm{F}, \mathrm{O}$, and $\mathrm{N}$ atoms in problem-solving is evaluated as a result of this heuristic. These students trusted in $\mathrm{H}, \mathrm{F}, \mathrm{O}$, and $\mathrm{N}$ atoms as they have already known them from earlier classes, and it was easy to bring them from memory. After analyzing the obtained data, it was determined that most of the students who employed this rule for solving the problem did this unconsciously and thus gave the wrong answer. Besides, a few of the students who used the rule for solving the problem gave a correct answer.

Some students unconsciously over-generalized some patterns and rules that they have learned before solving the problem. This situation is a result of the generalization. The student statements indicating the over-generalization of patterns of rules are as such: "Octet rule applies in the formation of hydrogen bond", "Hydrogen bonding is a chemical reaction (condensation)" and "The number of bonds is taken into consideration during the formation of hydrogen bond". The heuristic of generalization, which becomes evident in such reasoning, played an essential role in triggering other heuristics. The reasoning process of participant S4 presented above is an example of how generalization heuristic triggers other heuristics.

One of the participants stated that (S29) s/he made a project work about hydrogen bonding when $\mathrm{s} / \mathrm{he}$ was in high school, he liked doing the project, completed it successfully, got positive feedback from his/her teacher, and got a high score at the end of the project. S/he mentioned that s/he liked the subject, $\mathrm{s} /$ he has always been interested in it, and $\mathrm{s} /$ he has more than positive feelings about hydrogen bonding. Affect heuristic is encoded based on these statements of S29. Three of the students (S1, S8, S23) mentioned that they generally hated non-numerical chemistry classes, they felt close to the mentality of numerical classes, subjects in chemistry classes mostly require chemical and mathematical processes, they do not like abstract topics and relationships between these topics and as a result of this, they do not like the topic of hydrogen bonding. The students specifically stated that they are not interested in hydrogen bonding as it is a non-numerical chemistry field. These statements of S1, S8, and S23 are taken into consideration, and the affect heuristic is encoded based on them.

The ratio of students who gave correct answers to the question about hydrogen bonding is $16.66 \%$, which is a considerably low value. It was also determined that the correct answer ratio is similar to research in the literature about why reasoning processes in chemistry issues were similarly low. For example, according to research about "chemical bonding theories and molecular structures", the total correctness ratio of participator answers was 36\% (Ugras, 2018). Moreover, there is little research in the literature about chemistry topics with a high total correctness ratio of participator answers. For example, this 
ratio is $77 \%$ in the research analyzing acid strengths and student reasoning about the topic. Besides, although the correctness ratio was high in the research, it was determined that only less than $8 \%$ of students chose the correct answer based on scientific reasoning (McClary \& Talanquer, 2011). Except for Miller and Kim's research, none of the research about students' intuitive judgment in chemistry classes employed a model (such as the model of 10 heuristics in chemistry suggested by Talanquer) to classify heuristics. In their research, Miller and Kim used a form involving a structural demonstration of acetic acid to analyze the use of heuristic by students in hydrogen bonding. Miller and Kim handed the form to the participants and required them to write down their answers and explain why they preferred the answer. The researchers did not conduct interviews in their research; they solely analyzed the written answers of students. Because of this process, they stated that their study method was proper for determining 6 of the ten heuristics suggested by Talanquer; the researchers mentioned that their study was not proper for determining fluency rigidity, overconfidence, and effect (Miller \& Kim, 2017). As the most proper method for determining the heuristic use of students is the interview technique, specifically designed interviews were held with the participants to determine all of the heuristics suggested by Talanquer in this research study; this is why it was possible to determine the impacts of the entire ten heuristics suggested by Talanquer on students' reasoning processes in the subject of hydrogen bonding.

\section{CONCLUSION}

Cognitive science theories have been used to analyze individuals' judgments, decision-making processes, and reasoning types in these processes about daily life issues for years. Besides, there has been a very recent increase in the number of studies researching the impacts of heuristics, frequently mentioned in dual-process theory, on student reasoning in chemistry/science subjects. On the other hand, there is no study in the literature about how heuristics cause biases in students' judgment and decision making processes in chemistry/science subjects based on the interview data obtained from students in line with Talanquer's ten heuristics. In this respect, this research that evaluates and analyzes hydrogen bonding, a significant chemistry/science subject, will make a significant contribution to the literature in the context of cognitive psychology theory. Students' heuristics while answering the question of "Which atoms on acetic acid can form a hydrogen bond with water?" is determined for the first time in this study. Students commonly used the determined heuristics while answering the question indicates that most of them employed shorter strategies, precisely surface similarity, instead of scientific/chemical reasoning. Requiring a specific duty from students by presenting structural demonstration triggered the use of heuristic reasoning strategies. During the interviews, most of the students mentioned that they preferred the strategy that required the minimum amount of time. Participants who answered the question through the use of strategies based on the heuristics gave incorrect answers.

On the other hand, some students answered the question correctly by using heuristics. Students alone are not responsible for the frequent use of heuristic strategies; throughout their educational background, they are generally taught to use short-cut problem-solving strategies, which sometimes decrease their tendency to use cognitive ways or scientific reasoning skills in solving a problem. Because of this, they may have built the habit of solving problems by using short-cut strategies.

Intuitive judgment is one of the most common reasoning types. Educators' duty is not to prevent intuitive judgment; they are to research the impacts of this judgment on the understandings and interpretations of students, carefully analyze the data obtained at the end of the research process and form domain-specific, successful reasoning and articulation methods. While teaching a subject in General Chemistry classes, it might be advantageous to explain the possible incorrect reasoning encountered in using the common short-cut strategies in a specific subject. To ensure students have the habit of solving problems using chemical processes instead of short-cut strategies, which have nothing to do with scientific reasoning, it is suggested to require them to solve new and different types of chemistry questions. The question used in this research is an uncommon type for the participants. Although they encountered a different question, they frequently preferred to use familiar short-cut strategies; that is why we believe that there is a need to carry out more studies to determine which types of questions support students in terms of using chemical reasoning. It is indispensable to develop question types that encourage students to use chemical reasoning in chemistry questions with surface attributes such as structural demonstration. On the other hand, to correct the biases caused by Type 1 processes in different chemistry subjects, it is necessary to conduct more studies to determine how Type 2 processes can become more active.

There are various researches on students' understanding of intermolecular forces and their role in different chemical phenomena. All of these researches have focused on analyzing how students perceive the subject and phenomenon conceptually. However, it is also essential to determine how visually presented structural demonstrations affect students' conceptual understandings. At the end of this research, based on structural demonstration, it is determined that students overused the heuristics in solving the problem they were asked. This significant finding of the research should be considered; the fact that structural demonstrations trigger heuristics is once again revealed. This study shows that a multiple-select format assessment item can help 
understand how students evaluate hydrogen bonding. The use of open-ended interviews and a multiple-select format assessment item in this research has been highly beneficial for analyzing the relationship between structural demonstration and heuristic use.

This research reveals how different heuristics are efficient in students' reasoning processes about hydrogen bonding. This research will be beneficial for educators in determining strategies to prevent students' incorrect reasoning approaches. This research does not aim to decrease the use of heuristics or prevent the impacts of them; we suggest that it is also necessary to research different teaching strategies designed to decrease the negative impacts of heuristics, which are efficient during intuitive judgments.

\section{NOTES}

This article has been prepared from the relevant parts of Gülen Önal Karakoyun's doctoral dissertation. Gülen Önal Karakoyun's ORCID number: https://orcid.org/0000-0002-7675-0006. Erol Asiltürk's

ORCID number: https://orcid.org/0000-0001-81267812.

\section{REFERENCES}

Akerlind, G. S. (2005). Variation and commonality in phenomenographic research methods. Higher Education Research \& Development, 24(4), 321-334.

Barker, V., \& Millar, R. (2000). Students' reasoning about basic chemical thermodynamics and chemical bonding: what changes occur during a context-based post-16 chemistry course? International Journal of Science Education, 22(11), 1171- 1200.

Connor, P. E., \& Becker, B. W. (2003). Personal value systems and decision-making styles of public managers. Public Personnel Management, 32(1), 155-180.

Connor, M.C., Finkenstaedt-Quinn, S.A., \& Shultz, G.V. (2019). Constraints on organic chemistry students' reasoning during IR and $1 \mathrm{H}$ NMR spectral interpretation. Chemistry Education Research and Practice, 20, 522-541.

Cooper, M. M., Corley, L. M., \& Underwood, S. M. (2013). An investigation of college chemistry students' understanding of structure-property relationships. Journal of Research in Science Teaching, 50(6), 699-721.

Cooper, M. M., Williams, L. C., \& Underwood, S. M. (2015). Student understanding of intermolecular forces: a multimodal study. Journal of Chemical Education, 92(8), 1288-1298.

Didis N., Ozcan O., \& Abak A. (2008). Quantum physics from students' perspective: a qualitative study. Hacettepe Üniversitesi Egütim Fakëltesi Dergisi, 34, 86-94.

Duis, J. M. (2011). Organic chemistry educators' perspectives on fundamental concepts and misconceptions: An exploratory study. Journal of Chemical Education, 88(3), 346-350.

Evans, J. S. B. T. (2008). Dual-processing accounts of reasoning, judgment, and social cognition. Annual Review of Psychology, 59, $255-278$.

Evans, J. S. B. T., \& Stanovich, K. E. (2013). Dual-process theories of higher cognition: advancing the debate. Perspectives on Psychological Science, 8, 223-241.

Ferrari, J. R., \& Dovidio J. F. (2000). Examining behavioral processes in decision: decisional procrastination and decision-making style. Journal of Research in Personality, 34, 127-137.
Gigerenzer, G., \& Goldstein, D. G. (1996). Reasoning the fast and frugal way: models of bounded rationality. Psychological Review, 103, 650-669.

Gilovich, T., Griffin, D., \& Kahneman, D. (2002). Eds. Heuristics and Biases: The Psychology of Intuitive Judgment. Cambridge: Cambridge University Press.

Graulich, N. (2014). Intuitive judgments govern students' answering patterns in multiple-choice exercises in organic chemistry. Journal of Chemical Education, 92, 205-211.

Graulich, N., Hopf, H., \& Schreiner, P. R. (2011). Heuristic chemistryaddition reactions. Chemistry - A European Journal, 17, 30-40.

Graulich, N., Hopf, H., \& Schreiner, P. R. (2011). Heuristic chemistryelimination reactions. Chemistry - An Asian Journal, 6, 3180-3188.

Hastie, R. (2001). Problems for judgment and decision making. Annual Review of Psychology, 52, 653-683.

Henderleiter, J., Smart, R., Anderson, J., \& Elian, O. (2001). How do organic chemistry students understand and apply hydrogen bonding? Journal of Chemical Education, 78, 1126-1130.

Maeyer, J., \& Talanquer, V. (2013) Making predictions about chemical reactivity: assumptions and heuristics. J. Res. Sci. Teach. 50, $748-767$

Maeyer, J.R. (2013). Common-sense chemistry: the use of assumptions and beuristics in problem solving. PhD Thesis, Department of Chemistry and Biochemistry, The University of Arizona.

McClary, L. K., \& Talanquer, V. (2011). Heuristic reasoning in chemistry: making decisions about acid strength. International Journal of Science Education, 33, 1433-1454.

Miller, K., \& Kim, T. (2017). Examining student heuristic usage in a hydrogen bonding assessment. Biochemistry and Molecular Biology Education, 45(5), 411-416.

Pérez, J. R. B., Pérez, M. E. B, Calatayud M. L., García-Lopera R., Montesinos, J. V. S., \& Gil, E. T. (2017). Student's misconceptions on chemical bonding: a comparative study between high school and first year university students. Asian Journal of Education and eLearning, 5(1), 1-15.

Schwarz, N. (2004). Metacognitive experiences in consumer judgment and decision making. Journal of Consumer Psychology, 14(4), 332-348.

Stanovich, K. E., \& West, R. F. (2000). Individual differences in reasoning: implications for the rationality debate. Behavioral and Brain Sciences, 23(5), 645-726.

Taagepera, M., Arasasingham, R., Potter, F., Soroudi, A., \& Lam, G. (2002). Following the development of the bonding concept using knowledge space theory. Journal of Chemical Education, 79, 756-762.

Talanquer, V. (2014). Chemistry education: ten heuristics to tame. Journal of Chemical Education, 91, 1091-1097.

Tan, K. C. D., \& Treagust, D. F. (1999). Evaluating Students' Understanding of Chemical Bonding. School Science Review, 81, 7583.

Todd, P. M., \& Gigerenzer, G. (2000). Précis of simple heuristics that make us smart. Behavioral Brain Sciences, 23(5), 727-741.

Ugras, M. (2018). Heuristic reasoning of pre-service science teachers in chemistry topics. Journal of Baltic Science Education, 17(2), 343-356.

Vekli, G. S. (2020). Examination of prospective science teachers' levels of designing project directed to national support programs: A profile of Turkey. Journal of Science Learning, 3(2), 36-45.

Villafane, S. M., Bailey, C. P., Loertscher, J., Minderhout, V., \& Lewis, J. E. (2011). Development and analysis of an instrument to assess student understanding of foundational concepts before biochemistry coursework. Biochemistry and Molecular Biology Education, 39(2), 102-109.

Wihlborg, M. (2004). Student nurses' conceptions of internationalism in general and as an essential part of Swedish nurses' education. Higher Education Research \& Development, 23(4), 433-453. 